\title{
Hierarchical Organization of Parietofrontal Circuits during Goal-Directed Action
}

\author{
Lennart Verhagen, ${ }^{1,2} \mathrm{H}$. Chris Dijkerman, ${ }^{2}$ W. Pieter Medendorp,${ }^{1}$ and Ivan Toni ${ }^{1}$ \\ ${ }^{1}$ Donders Institute for Brain, Cognition, and Behavior, Radboud University Nijmegen, $6500 \mathrm{HB}$, Nijmegen, The Netherlands, and ${ }^{2}$ Experimental Psychology, \\ Helmholtz Institute, Utrecht University, 3508 TC, Utrecht, The Netherlands
}

Two parietofrontal networks share the control of goal-directed movements: a dorsomedial circuit that includes the superior parietooccipital sulcus (sPOS) and a dorsolateral circuit comprising the anterior intraparietal sulcus (aIPS). These circuits are thought to independently control either reach and grip components (a functional dissociation), or planning and execution phases of grasping movements (a temporal dissociation). However, recent evidence of functional and temporal overlap between these circuits has undermined those models.

Here, we test an alternative model that subsumes previous accounts: the dorsolateral and dorsomedial circuits operate at different hierarchical levels, resulting in functional and temporal dependencies between their computations. We asked human participants to grasp a visually presented object, manipulating movement complexity by varying object slant. We used concurrent single-pulse transcranial magnetic stimulation and electroencephalography (TMS-EEG) to probe and record neurophysiological activity in the two circuits. Changes in alpha-band oscillations $(8-12 \mathrm{~Hz})$ characterized the effects of task manipulations and TMS interferences over aIPS and sPOS.

Increasing the complexity of the grasping movement was accompanied by alpha-suppression over dorsomedial parietofrontal regions, including sPOS, during both planning and execution stages. TMS interference over either aIPS or sPOS disrupted this index of dorsomedial computations; early when aIPS was perturbed, later when sPOS was perturbed, indicating that the dorsomedial circuit is temporally dependent on aIPS. TMS over sPOS enhanced alpha-suppression in inferior parietal cortex, indicating that the dorsolateral circuit can compensate for a transient sPOS perturbation. These findings suggest that both circuits specify the same grasping parameters, with dorsomedial computations depending on dorsolateral contributions.

\section{Introduction}

The control of grasping movements relies on two parietofrontal circuits: a dorsolateral and a dorsomedial pathway (Grafton, 2010). The dorsolateral circuit includes the anterior intraparietal sulcus (aIPS) and the ventral premotor cortex (PMv); the dorsomedial circuit connects the superior parieto-occipital sulcus (sPOS) and the dorsal premotor cortex (PMd). These two circuits have been suggested to control either different motoric functions in parallel (Jeannerod et al., 1995; Culham et al., 2006; Vesia and Crawford, 2012) (e.g., reaching and grasping components), or different temporal phases of an action, serially (Pisella et al., 2000; Rizzolatti and Matelli, 2003; Glover, 2004) (e.g., planning and on-line control). Here, we test the hypothesis that these two circuits support the same functional components of grasping, in

Received Aug. 16, 2012; revised Feb. 26, 2013; accepted March 1, 2013.

Author contributions: L.V., H.C.D., and I.T. designed research; L.V. performed research; L.V. analyzed data; L.V., H.C.D., W.P.M., and I.T. wrote the paper.

This work was supported by The Netherlands Organization for Scientific Research: Open Competition Grant No. 400-04-379 (H.C.D., I.T.), VICI Grant No. 453-08-002 (I.T.), VICI Grant No. 453-10-003 (H.C.D.), European Research Council Grant No. 263567 (W.P.M.), and the BrainGain Smart Mix Programme of The Netherlands Ministry of Economic Affairs/Ministry of Education, Culture and Science (I.T.). We thank Inge Leunissen for her assistance with data acquisition; and Ole Jensen, Luc Selen, and Tim van Maurik for their advice on methodological issues.

The authors declare no competing financial interests.

Correspondence should be addressed to Dr. Lennart Verhagen, Radboud University Nijmegen, PO Box 9101, 6500 HB, Nijmegen, The Netherlands. E-mail: lennart.verhagen@donders.ru.nl.

DOI:10.1523/JNEUROSCI.3928-12.2013

Copyright $\odot 2013$ the authors $\quad 0270-6474 / 13 / 336492-12 \$ 15.00 / 0$ parallel, but with serial temporal dependencies, which is indicative of a hierarchical organization.

This hypothesis is grounded on two sets of observations. First, the two circuits, which are monosynaptically connected (Borra et al., 2008; Gamberini et al., 2009; Gerbella et al., 2011; Gharbawie et al., 2011), show functional overlap in the processing of intrinsic object features, such as size and shape, to plan a grasping movement (Murata et al., 2000; Fattori et al., 2010). Second, there is evidence that the operations of the two circuits have different temporal profiles (Tunik et al., 2008). TMS interferes earlier with dorsolateral than with dorsomedial contributions (Davare et al., 2006; Taubert et al., 2010), and aIPS rapidly builds a preliminary motor plan based on multiple sources of information (Verhagen et al., 2012). Yet, it remains to be seen how the functions and temporal dynamics of these two circuits interact. This interaction can only be studied when their contributions are separately probed and directly contrasted.

We address this issue by using a combination of movement analyses, EEG, TMS, and task manipulations requiring human subjects to grasp objects oriented at different slants. The slant of the object influences the sensorimotor complexity of the movement: a horizontally slanted objects requires a more complex grasp than a vertically slanted object because it is harder to reliably reach the digit endpoints and to accommodate tighter spatial constraints (Gentilucci et al., 1996; van Bergen et al., 2007; Brouwer et al., 2009). Furthermore, both BOLD and EEG power mea- 
A experimental setup

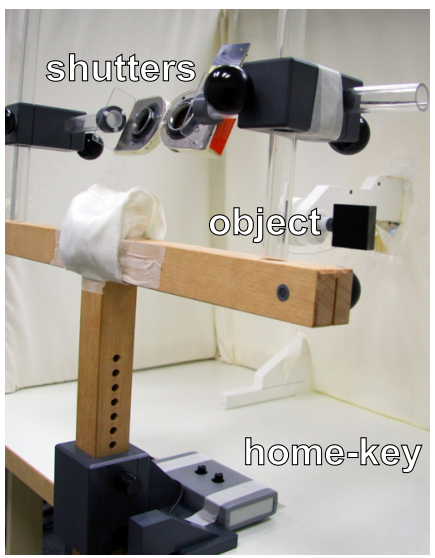

B TMS sites

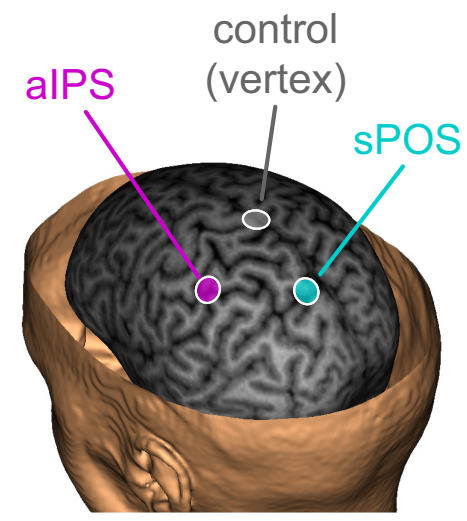

C experimental manipulation

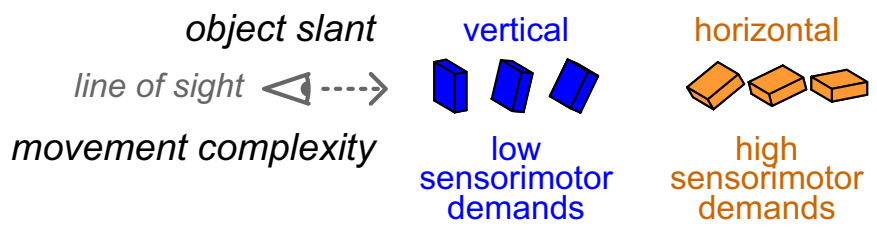

D trial timecourse

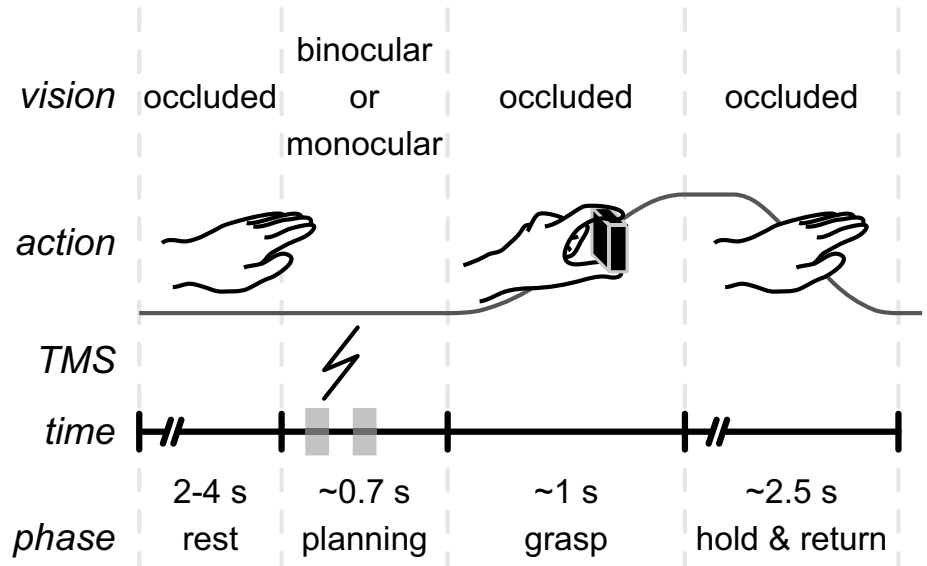

Figure 1. Experimental setup and task. $A$, The subject was seated at a table with his or her head resting in a chin-rest, the right hand on a home-key, and vision occluded by two shutters. These shutters could open individually to allow vision of the object to be grasped (a $6 \times 6 \times 2 \mathrm{~cm}$ black rectangular object), which was suspended in front of the subject. $\boldsymbol{B}$, A TMS coil was positioned over the subject's head targeted at one of three sites: (1) the anterior part of the left intraparietal sulcus (magenta, alPS), (2) the anterior bank of the superior part of the parieto-occipital sulcus in the left hemisphere (cyan, SPOS), and (3) the vertex of the head (gray) serving as a control site. TMS target sites are projected on a curvilinear reconstruction of the structural MRI of one exemplary subject, shown here at $6 \mathrm{~mm}$ in depth from the cortical surface. The location of the aIPS and sPOS sites were based on group averaged fMRI activation coordinates from a previous study (Verhagen et al., 2008). C, To vary the sensorimotor demands of the grasping task we manipulated the slant of the target object along the midsagittal plane: in seven slants from vertical to horizontal relative to the frontoparallel plane, in $15^{\circ}$ steps. $\boldsymbol{D}$, At the start of each trial, after a random interval $(2-4 \mathrm{~s})$ with occluded vision, either monocular or binocular vision of the target object was provided. The subject was instructed to prepare and perform a grasping movement, displacing the target object before returning to the starting position. During the planning phase, a single TMS pulse was delivered between either $100-200 \mathrm{~ms}$ or $300-400 \mathrm{~ms}$ after stimulus presentation (indicated on the trial time course with gray blocks). As soon as the subject released the home-key to grasp the object, the shutter glasses closed, preventing visual feedback of the movement.

sures have shown that this grasping task engages both dorsolateral and dorsomedial circuits (Verhagen et al., 2008, 2012). In this study, functional and temporal dependencies between dorsolateral and dorsomedial processes were assessed by perturbing neural processing in either aIPS or sPOS with single-pulse TMS during grasp preparation. The consequences of those TMS perturbations were quantified behaviorally with movement analyses, and cerebrally by tracking changes in alpha-band and beta-band oscillatory power, well known indices of increased computational load (Neuper et al., 2006; Jensen and Mazaheri, 2010). The sensitive measurements provided by the integration of these techniques indicate that both parietofrontal circuits support the same functional components of grasping, in parallel, with the dorsomedial circuit serially dependent on a preliminary motor plan structured by the dorsolateral circuit.

\section{Materials and Methods}

Subjects

The dataset used in this study was acquired in the context of a larger dataset, parts of which were described previously (Verhagen et al., 2012). These studies used the same cohort of subjects, applying the same inclusion and exclusion criteria and basic data preprocessing steps, but report different sets of results. In this report, we summarize the overlapping methodology, and describe in detail the novel aspects of this study. Datasets from 24 healthy, right-handed subjects (mean \pm SD, age: $21 \pm 2$ years; handedness: $89 \pm 11 ; 9$ males) (Oldfield, 1971) with normal or corrected-to-normal vision were included in this experiment. The study was approved by the local ethics committee and a written informed consent was obtained before the start of the experiment according to the Declaration of Helsinki.

\section{Experimental setup and task}

Subjects were seated at a table; their head was stabilized by a chin rest. They wore ears plugs, and their right hand rested on a button box, pressing down the "home-key." Vision was controlled by two mechanical shutters that could be opened and closed independently within $3.4 \mathrm{~ms}$ (Fig. 1A). At the start of a trial, subjects' vision was occluded. Then, after a random time interval (2-4 s), either one or both shutters opened, allowing monocular or binocular vision of the object to be grasped. The target object (a black rectangular object, $6 \times 6 \times 2 \mathrm{~cm}$ ) was displayed against a white background, suspended in front of the subject along the midsagittal plane at a comfortable reaching distance $(\sim 25 \mathrm{~cm}$ in front and $\sim 30$ $\mathrm{cm}$ above the starting position of the right hand) (Fig. 1A). The orientation of the object could be varied around its pitch axis, in seven steps of $15^{\circ}$ between the frontoparallel (vertical) and transversal (horizontal) plane. In the vertical configuration $\left(0^{\circ}\right.$ relative to the frontal plane) the object is suspended upright with its largest surface facing the subjects directly. Subjects grasped the vertical object with the index finger on the top of the object and the thumb on the bottom. When the slant of the object is increased, the top of the object is further away from the subjects (and the bottom closer). In the horizontal configuration $\left(90^{\circ}\right.$ relative to the fron- 
tal plane) the object's largest surface is in parallel with the tabletop. Subjects grasped the horizontal object with the index finger on the far end of the object and the thumb on the close end. They slid the object from its support, held it in mid-air, placed it back into its support, and then returned to the starting position (Fig. $1 B$ ). As soon as the subjects started to grasp (releasing the home-key), their vision was occluded. This absence of visual feedback, as well as the requirements to hold the object against gravity, further ensured that the subjects prepared an accurate grasping movement.

A trial lasted between 6 and $11 \mathrm{~s}$ (mean, $\sim 8 \mathrm{~s}$ ). Subjects performed 6 blocks of 84 trials each ( $\sim 11$ min per block), a total of 504 trials for the whole experiment ( $\sim 60$ min including short breaks between each block).

\section{Experimental design}

We considered two factors: TMS site (three levels: control, aIPS, and sPOS) (Fig. $1 B$ ), and object slant (two levels: vertical: $0^{\circ}, 15^{\circ}, 30^{\circ}$; horizontal: $60^{\circ}, 75^{\circ}, 90^{\circ}$ ) (Fig. $1 C$ ). This experimental design manipulates the demands on visuospatial transformations by varying object slant, testing the effects of TMS over aIPS and sPOS. With increasing object slant, the wrist, thumb, and especially the index finger need to be lifted and transported further along a more constrained path requiring increased coordination of multiple joints. The end-point of the index finger becomes occluded and the object becomes an obstacle on the intended trajectory for the hand and fingers. These constraints increase the demands for accurate sensorimotor transformations. If both aIPS and sPOS contribute to these sensorimotor processes, then TMS over either region is expected to disturb an electrophysiological correlate of these processes, i.e., suppression of low-frequency cerebral oscillation (Verhagen et al., 2012). We can track the dynamics of these changes in oscillatory power by virtue of the temporal specificity of EEG.

To determine whether the expected modulations were driven by the required output of the system, or alternatively by its available input, we considered the type of vision of the object as a post hoc factor (two levels: binocular, monocular). Binocular (stereoscopic disparity) and monocular (pictorial) cues of depth are differentially dependent on the slant of the object, with binocular cues being most informative for vertically slanted objects, and monocular cues for horizontally slanted objects (Knill, 2005). If the sensorimotor transformations are fully determined by the quality of the visual cues they should follow the differential dependency of vision on object slant (i.e., reveal a different relationship between parietofrontal circuit involvement and object slant for monocular and binocular trials). However, if the sensorimotor transformations are only informed by the required movement, the increasing demands for accurate sensorimotor transformations with increasing object slant should be equal for monocular and binocular trials.

The seven object slants were binned in two factorial levels of object slant (vertical: $0^{\circ}, 15^{\circ}, 30^{\circ}$; horizontal: $60^{\circ}, 75^{\circ}, 90^{\circ}$ ) to maximize the number of trials per condition for statistical inference (see below, EEG: statistical inference). We arbitrarily chose to provide monocular vision to only the right eye. To prevent a preference by expectation for the right eye we included left eye monocular trials as catch trials ( $~ 8 \%$ of the total). In summary, each condition of the TMS site $(3) \times$ slant $(2) \times$ vision $(2)$ design contained 33 trials; a total of 396 trials were included in the analysis, and 108 additional trials were considered catch trials (66 trials with objects slanted at $45^{\circ}$, and 42 trials allowing only monocular vision to the left eye). Experimental conditions were pseudorandomized and evenly distributed across trials.

\section{TMS}

On each trial, we delivered a single monophasic TMS pulse (Magstim) at a randomized time point between 100 and $400 \mathrm{~ms}$ after stimulus presentation (but before movement onset). This relatively wide temporal range allowed inferences to be drawn from the whole preparation phase, but minimized the predictability of the TMS occurrence for the subjects. No TMS pulses were delivered between 200 and $300 \mathrm{~ms}$ after stimulus presentation to ensure that a sufficient number of trials could contribute to a continuous averaged EEG signal unaffected by TMS artifacts. The pulses were delivered at a particularly low intensity, at the active motor threshold (AMT) (Rossini et al., 1994), improving functional specificity (Verhagen et al., 2012). Average AMT was $33 \pm 7 \%$ (mean \pm SD) of maximum stimulator output. Subjects wore earplugs during both AMT determination and the experimental sessions to minimize the acoustic effect of TMS delivery.

During each of the six blocks of trials, TMS was applied with the coil centered over one of three different sites, counterbalanced within subjects: (1) group-averaged coordinates of the left anterior region of the intraparietal sulcus (Verhagen et al., 2008), the putative human homolog of the macaque anterior intraparietal area (Culham et al., 2006) (2) group-averaged coordinates of the anterior bank of the superior part of the parieto-occipital sulcus in the left hemisphere (Verhagen et al., 2008), the putative human homolog of macaque visual area $6 \mathrm{~A}$ (Cavina-Pratesi et al., 2010), or (3) a site controlling for nonspecific TMS effects (the vertex of the head). The coil was oriented in such a way that first half of the monophasic pulse was delivered over the aIPS site in an inferior/ posterior to superior/anterior direction, over the sPOS site from inferior to superior direction, and over the vertex of the head from a posterior to anterior direction. Continuous online stereotactic guidance of the TMS coil was physically incompatible with the current experimental setup. Therefore, we adopted an alternative approach to ensure anatomical and functional consistency in the TMS intervention (described in detail in a previous report) (Verhagen et al., 2012). In brief, by using a low-intensity stimulation we ensured that only neuronal populations activated by the task will be effectively perturbed, whereas other neuronal populations with a similar distance from the TMS coil, but not activated by the task remain functionally unaffected task (Allen et al., 2007). Furthermore, preceding the data acquisition in the main cohort of subject, we quantified the variability of TMS site localization in six additional pilot subjects. Specifically, we transformed the stereotactic coordinates of aIPS and sPOS (MNI coordinates: [ $-42-4442]$ and [ $-18-7250]$ ) (Verhagen et al., 2008) into the native anatomical space of each pilot subject (as defined by their structural MRI scan). The projection of that location on each subject's scalp did not vary across the pilot group $>7 \mathrm{~mm}$ for the aIPS site and $9 \mathrm{~mm}$ for the sPOS site. This variability is within the spatial range of the effective magnetic field changes induced by the TMS pulse and within the variability achieved using alternative neuronavigation approaches (Sack et al., 2009). We mapped the aIPS and sPOS sites (Fig. $1 B$ ) off-line with respect to the 10-10 system for EEG electrode positioning after the procedure described in detail in a previous report. The scalp-projection of the aIPS site fell near electrode CP3, superior to electrode CP5 located above the supramarginal gyrus (Koessler et al., 2009). The scalp-projection of the sPOS site fell just left of the midpoint between electrodes $\mathrm{POz}$ and $\mathrm{Pz}$. The vertex site, serving as a control site, was defined as the point where both the sagittal midline from nasion to inion and the coronal line from ear to ear were dissected in the middle (Jasper, 1958; Chatrian et al., 1985).

\section{Kinematics}

Data acquisition. We sampled position and orientation of four sensors at $250 \mathrm{~Hz}$, using an electromagnetic tracking system (LIBERTY, Polhemus). Three sensors were placed on the hand and fingers: on the nail of the thumb, the nail of the index finger, and on top of the first metacarpophalangeal joint (labeled "hand"). The fourth sensor was positioned on the object to be grasped, along its axis of rotation.

Data analysis. Kinematic data were analyzed off-line using MATLAB (Mathworks). A detailed specification of the analysis procedure can be found in Verhagen et al. (2012).

First, we preprocessed the sensor time series: removing the TMSinduced artifact, low-pass filtering at $15 \mathrm{~Hz}$ (sixth order Butterworth), and defining a virtual "grip" sensor as the mean position of the thumb and index finger sensors. We then calculated several derivatives: velocity, acceleration, grip aperture, grip orientation, and grip velocity.

Subsequently, we took particular care to robustly estimate the onset and offset of the movement. We used a multiple-sources of information approach, formalized in both binary and continuous functions weighted to find optimal points of transition (Schot et al., 2010). Movement onset was defined as the point in time when the fingers just started moving (binary: velocity $>0.02 \mathrm{~m} / \mathrm{s}$ ), but their velocity and the grip aperture were as small as possible (continuous: velocity $\downarrow 0$, aperture $\downarrow 0$ ). To identify movement offset, the grip sensor must be and stay close to the object 
(binary: distance $<7 \mathrm{~cm}$ ), grip velocity must be decreasing and stay low (binary: grip velocity $<0.05 \mathrm{~m} / \mathrm{s}$ ), whereas the velocities of the sensors and the grip, and the grip aperture must be as small as possible (continuous: velocity $\downarrow 0$, aperture $\downarrow 0$ ).

To characterize the kinematics of the grasping movement we calculated commonly used kinematic parameters (Jeannerod, 1984): reaction time (RT), movement duration (MT), trajectory length (TL), mean velocity $(\mu \mathrm{V})$, mean transport velocity (during first $75 \%$ of the movement; $\mu \mathrm{TV}$ ), mean approach velocity (during last $25 \%$ of the movement; $\mu \mathrm{AV}$ ), peak velocity $(\mathrm{PV})$, time to peak velocity (tPV), peak grip aperture (PGA), time to peak grip aperture (tPGA). The TL, $\mu \mathrm{V}, \mu \mathrm{TV}, \mu \mathrm{AV}, \mathrm{PV}$, and $\mathrm{TPV}$ parameters were based on the velocity time series of the virtual grip sensor, i.e., the mean of the thumb and index finger sensors.

Finally, we described the spatial variability of the movements of the hand, thumb and index finger by calculating spatial confidence intervals of the trajectories at multiple points along the movement. These spatial confidence intervals were obtained separately for each object slant in each individual subject based on spatially averaged trajectories. Our approach is similar to the method described by Liu and Todorov (2007) but resulted in $2 \mathrm{D}$ confidence ellipses for each point along the trajectory in a plane perpendicular to the movement direction at that point. Averaging trajectories in space, regardless of time, is not trivial and requires an iterative algorithm to converge upon a solution (Liu and Todorov, 2007). To initialize the cycle, all movements were resampled to 1000 points on equidistant intervals along the trajectory. An average trajectory was created by taking the mean of all trajectories on a point-by-point basis. We then restructured the individual trajectories by taking the closest point from each resampled 1000-point trajectory to each point of the average. To minimize the chance that one point of an individual trajectory maps onto multiple points of the average, the average was first down-sampled to 300 equidistant points. This cycle of steps was repeated with the restructured trajectories being used to calculate a new average in an iterative fashion until convergence upon a stable spatially averaged trajectory (i.e., no change from one iteration to the next). The trajectory variability was calculated on basis of these resampled trajectories. For each of the 300 points of the average, the instantaneous gradient in threedimensions was used as a normal vector to describe a plane perpendicular to the movement direction. For all trajectories the intersection with this plane was calculated using linear interpolation resulting in a $2 \mathrm{D}$ distribution of points (oriented in 3D). The confidence ellipse was calculated on the basis of an Eigen-decomposition of the covariance matrix of this set of points and scaled using a $\chi^{2}$ distribution to match a $95 \%$ confidence interval in 2D (McIntyre et al., 1998). The critical parameter describing the variability of the trajectories along the movement is the area of the confidence ellipses resulting in 300-point time-series.

Statistical inference. We excluded trials in which subjects started moving before or at the time of the TMS pulse, and trials where the main kinematic parameters deviated from the first or third quartile by more than three interquartile ranges. On average, $88 \pm 5 \%$ (mean \pm SD) of all trials passed the exclusion criteria and entered further analysis. Statistical inferences on the kinematic parameters described above (RT, MT, TL, $\mu \mathrm{V}, \mu \mathrm{TV}, \mu \mathrm{AV}, \mathrm{PV}, \mathrm{tPV}, \mathrm{PGA}, \mathrm{tPGA})$ were drawn using the SPSS software package 16.0 (SPSS). All parameters were checked on skewness and kurtosis, and found to comply with the assumption of normal distribution. Trials were averaged for each experimental condition and subject; the resulting means were entered in a univariate repeated-measures ANOVA considering the factors TMS site (3), object slant (2), and vision (2) testing for effects between conditions within subjects.

Statistical inference on the spatial variability of the movements of the hand, thumb, and index finger was drawn using a nonparametric approach to accommodate the inherent autocorrelation of the data series, similar to the statistical inference of the EEG data (see below, EEG: statistical inference). In accordance with the analysis of the other kinematic parameters we averaged the area of the $95 \%$ confidence ellipses of the three most vertical configurations $\left(0^{\circ}, 15^{\circ}, 30^{\circ}\right)$ and the three most horizontal configurations $\left(60^{\circ}, 75^{\circ}, 90^{\circ}\right)$, resulting in a two-level factor of object slant. Within each subject, trajectory-points were identified for which the difference between conditions exceeded an arbitrary threshold $(p<0.05)$ in an independent samples $t$ test. A cumulative statistic was calculated for each cluster of surviving points connected along the trajectory. An accurate Monte Carlo estimate of the permutation $p$ value is obtained by comparing the cluster-level statistic of interest to the distribution of cluster-statistics calculated from 10,000 random permutations of the conditions.

\section{EEG}

Data acquisition. We recorded the EEG using $31 \mathrm{Ag} / \mathrm{AgCl}$ electrodes, organized on a flat-tip cap (BrainProducts) according to the 10-10 system (Chatrian et al., 1985). Electrical voltage was sampled at $5000 \mathrm{~Hz}$ using an amplifier with a high dynamic range and capable of direct current recording (MR+DC BrainAmp, BrainProducts). The EEG data were low-pass filtered at $1000 \mathrm{~Hz}$. Electrical artifacts associated with the TMS pulse were minimized during acquisition by the use of tip, rather than classical circular, electrodes and by positioning the EEG cables perpendicular to the orientation of the TMS coil whenever possible. Furthermore, to minimize the temporal spread of TMS induced artifacts, no high-pass filter was applied at acquisition.

Data analysis. After excluding trials with prominent eye movements, blinks, and muscle artifacts (on the basis of visual inspection of the data), EEG data were processed and analyzed using a MATLAB toolbox (FieldTrip, http:www.ru.nl/neuroimaging/fieldtrip/) (Oostenveld et al., 2011). TMS artifacts were identified, modeled, and removed using an iterative least-squares optimization algorithm when possible, or otherwise the trial was discarded (Ilmoniemi and Kicić, 2010; Verhagen et al., 2012). On average, $79 \pm 6 \%$ (mean \pm SD. across subjects) of the trials survived both the kinematic and EEG exclusion criteria. The remaining data were re-referenced to the average signal of all sensors to remove any spatial effects on voltage differences with respect to the location of the reference electrode. By means of independent component analysis (Lee et al., 1999; Makeig et al., 2004) residual signals related to eye movements, blinks, muscle tension, and TMS artifacts were identified and removed (Korhonen et al., 2011). After power line noise was removed (using discrete Fourier transform notch filters at 50,100, and $150 \mathrm{~Hz}$ ), data were bandpass filtered $(0.75-150 \mathrm{~Hz}$ using a sixth order Butterworth filter) and down-sampled to $500 \mathrm{~Hz}$.

For each trial and sensor we calculated time-frequency representations (TFRs) of oscillatory power using a Fourier transform approach $(8-35 \mathrm{~Hz}$, in steps of $1 \mathrm{~Hz}$ ) applied to sliding time windows (sliding in 20 ms steps) multiplied by a Hanning taper. We focused on a low-frequency range $(8-35 \mathrm{~Hz})$ where power can be robustly estimated with minimal signal deterioration by potential artifacts. In this range, two prominent and dissociable oscillatory rhythms are present that index cerebral activity: the alpha $(9-12 \mathrm{~Hz})$ and beta $(18-24 \mathrm{~Hz})$ frequency bands. We performed the time-frequency analyses with two different time-window durations: a relatively short $200 \mathrm{~ms}$ taper ( $5 \mathrm{~Hz}$ frequency smoothing), and a more conventional $400 \mathrm{~ms}$ taper $(2.5 \mathrm{~Hz}$ frequency smoothing). The $200 \mathrm{~ms}$ taper was used when estimating oscillatory power shortly before and after the TMS pulse, prioritizing temporal above spectral specificity. Power could not be estimated in a 220 or $420 \mathrm{~ms}$ window around the TMS pulse, for the 200 and $400 \mathrm{~ms}$ tapers, respectively (the width of one taper and one additional step of $20 \mathrm{~ms}$ ). However, because of the trial-to-trial variation in the time of the TMS pulse, we could still generate a continuous average estimate of power using the short taper. For each experimental condition, power estimates were averaged over trials, log-transformed, and related to a baseline period from the same trials (relative change from $[-700-200] \mathrm{ms}$ before stimulus presentation). Subsequently, the individual effects were grand averaged over subjects to estimate the group effects.

Statistical inference. Within each subject, the difference between conditions was quantified as the difference of the relative log-transformed mean power changes. Statistical inference $(p<0.05)$ was performed at the group level (within-subjects random-effects analysis) using a nonparametric randomization test controlling for multiple-comparisons across the sensor, frequency, and time dimensions (SFT points) (Maris and Oostenveld, 2007). This nonparametric test requires two conditions to be randomly swapped, preventing a full factorial analysis similar to the ANOVA of the behavioral data. Importantly, in the current experimental design the factors TMS site, object slant, and vision were orthogonal to 
each other. Given that the nonparametric permutation approach estimates a permutation $p$ value based on the actual data, it is not necessary to parametrically model the effects of all experimental factors simultaneously to derive an accurate estimate of the true $p$ value. The nonparametric permutation test consisted of several steps. First, the conditions of interest are described for each subject independently by either the average of the corresponding trials, or in case of a within-subject contrast by the difference between condition averages. Second, SFT points are identified for which the $t$ values for the difference between conditions over subjects exceed an arbitrary threshold (independent samples $t$ test, $p<0.05$ ). Third, surviving SFT points are grouped in clusters, defined by their continuity in one or multiple dimensions. The $t$ values from each SFT point in a cluster are added, and this cumulative statistic is used for inferential statistics at the cluster level. Fourth, a Monte Carlo estimate of the permutation $p$ value of the cluster is obtained by comparing the clusterlevel test statistic with a randomization null distribution assuming no difference between conditions. This distribution is obtained by randomly swapping the conditions within subjects and repeating the steps above to calculate the cluster-level test statistic 10,000 times, resulting in an accurate Monte Carlo estimate of the true $p$ value.

\section{Results}

We investigated the causal, temporal, and functional dependencies between the dorsolateral and dorsomedial parietofrontal circuits supporting grasping movements. We manipulated the demands on sensorimotor processing in these circuits by changing the slant of an object to be grasped, varying the difficulty of movement specification. Using TMS, we perturbed the neural computations of aIPS (in the dorsolateral circuit) and those of sPOS (in the dorsomedial circuit). We assessed both the behavioral and cerebral consequences of the task and of the TMS intervention, as indexed by kinematic measurements and by modulations in spectral power in the alpha and beta bands, as recorded by EEG.

\section{Behavioral effects of grasping a slanted object: increased sensorimotor demands change grasping kinematics}

Behavioral measurements obtained after control TMS over the vertex indicated that grasping a horizontal object places higher demands on sensorimotor processing than grasping a vertical object. When grasping a vertically oriented object the target endpoints of the thumb and index finger can be reached relatively straightforwardly. In contrast, grasping a horizontally oriented object involves satisfying two additional constraints: (1) specifying an unseen endpoint for landing the index finger on the object while avoiding collision with its distal edge; and (2) moving the index finger along a longer path over the object while the wrist is being flexed (Gentilucci et al., 1996; Mamassian, 1997; van Bergen et al., 2007; Brouwer et al., 2009). The first constraint is meant to reduce the velocity and variability of the trajectory of the index finger when it closely approaches the desired end-point, as required by the need to avoid collisions with the object edge (acting both as an obstacle and a target) and slippage. The second constraint is meant to increase the variability of the trajectory of both fingers and the hand when the hand is approaching a horizontally oriented object. These qualitative hypotheses were confirmed in a quantitative comparison of the spatial variability of the movement trajectories. More specifically, we indexed the spatial distribution of the hand, thumb, and index finger at multiple points along the trajectory by estimating $95 \%$ confidence ellipses perpendicular to the movement direction (Fig. $2 A, B$ ). The spatial variability of the trajectory is described by the evolution of the area of these confidence ellipses across the trajectory, separately for grasping horizontally oriented and vertically oriented objects (Fig. 2C-E). It can be seen that, during the final 5\% of the movement, the variability of the index finger is significantly reduced when grasping horizontally oriented objects $(p=0.012)$ (Fig. $2 C)$. This effect occurs when the index finger needs to curl around the edge of the object (Fig. 2B). This finding supports the notion 


\section{Behavioral effects of varying sensorimotor demands of grasping (object slant)}
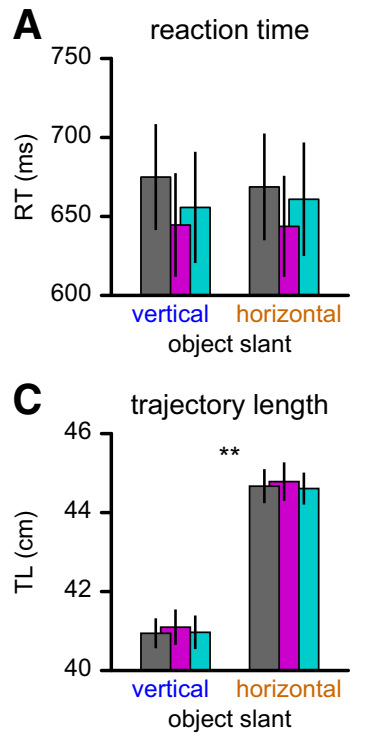
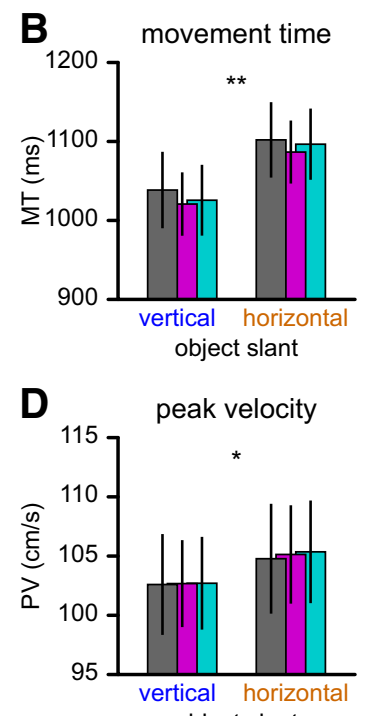

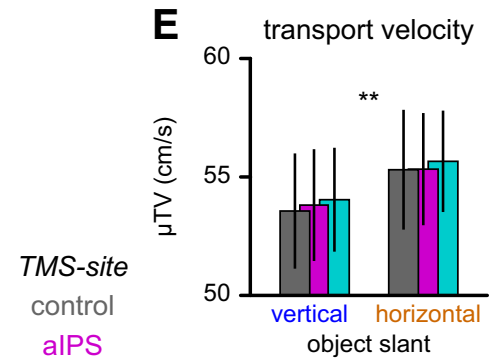

sPOS

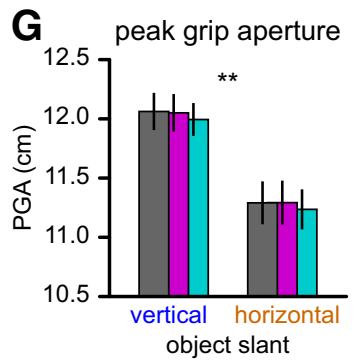

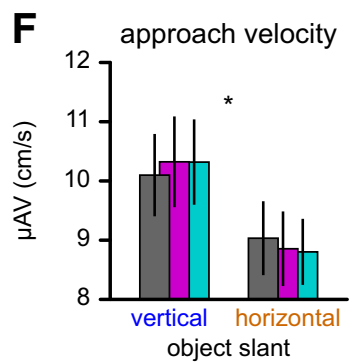

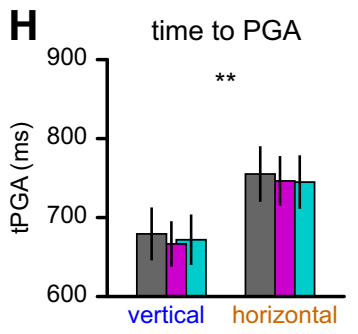

object slant

Figure 3. Behavioral effects of varying sensorimotor demands of grasping by object slant. $\boldsymbol{A}-\boldsymbol{F}$, Bar plots showing kinematic parameter values of the effect of object slant (mean \pm SEM). Color coding is as in Figure 1, B and C: bars are grouped by object slant (blue, vertical; orange, horizontal), with bar face color indicating the site of TMS (gray, control; magenta, alPS; cyan, sP0S). Significant effects of object slant are indicated with stars $\left({ }^{*} 0.01<p<0.05 ;{ }^{* *} p<0.001\right)$. A, RTs were not modulated by object slant, but were significantly shorter after TMS over alPS compared with control stimulation. $\boldsymbol{B}$, Grasping movement durations increased with increasing object slant. $\boldsymbol{C}, \boldsymbol{D}$, The increase in movement duration was driven by an increased TL and accompanied by a small increase in their PV.E, F, During the transport toward the object (first $75 \%$ of the movement) the velocity of the virtual grip sensor increased with increasing object slant ( $\mu$ TV). In contrast, when approaching near horizontal objects (last $25 \%$ of the movement) the fingers moved with a slower velocity than when approaching near vertical objects ( $\mu$ AV). $\mathbf{G}, \boldsymbol{H}$, The PGA was smaller and occurred later (tPGA) when grasping more horizontally oriented objects.

Table 1. Behavioral effects

\begin{tabular}{|c|c|c|c|c|c|c|c|}
\hline \multirow[b]{2}{*}{ Parameter } & \multirow[b]{2}{*}{ Effect of slant ${ }^{a}$} & \multicolumn{2}{|c|}{ Control TMS $^{b}$} & \multicolumn{2}{|c|}{ TMS over alPS $^{b}$} & \multicolumn{2}{|c|}{ TMS over sPOS $^{b}$} \\
\hline & & Vertical & Horizontal & Vertical & Horizontal & Vertical & Horizontal \\
\hline RT (ms) & $0.537(0.39)$ & $672(34)$ & $669(35)$ & $642(33)$ & $644(33)$ & $653(36)$ & $661(37)$ \\
\hline $\mathrm{MT}$ (ms) & $0.000(30.26)$ & $1038(49)$ & 1101 (49) & $1020(41)$ & $1086(41)$ & $1026(46)$ & 1097 (46) \\
\hline $\mathrm{TL}(\mathrm{cm})$ & $0.000(336.58)$ & $40.9(0.4)$ & $44.7(0.4)$ & $41.1(0.5)$ & $44.8(0.5)$ & $41.0(0.4)$ & $44.6(0.4)$ \\
\hline$\mu \mathrm{V}(\mathrm{cm} / \mathrm{s})$ & $0.029(5.46)$ & 41.9 (1.9) & $42.8(2.0)$ & $42.3(1.8)$ & $42.9(1.6)$ & $42.2(1.9)$ & 42.7 (1.8) \\
\hline$\mu \operatorname{TV}(\mathrm{cm} / \mathrm{s})$ & $0.001(14.69)$ & $53.5(2.4)$ & $55.4(2.2)$ & $53.8(2.4)$ & $55.4(2.4)$ & $54.1(2.2)$ & $55.7(2.1)$ \\
\hline$\mu \mathrm{AV}(\mathrm{cm} / \mathrm{s})$ & $0.019(6.42)$ & $10.1(0.7)$ & $9.1(0.6)$ & $10.4(0.8)$ & $9.1(0.6)$ & $10.3(0.7)$ & $8.8(0.6)$ \\
\hline $\mathrm{PV}(\mathrm{cm} / \mathrm{s})$ & $0.029(5.48)$ & $102.5(4.3)$ & $104.9(4.8)$ & $102.8(3.8)$ & $105.1(4.3)$ & $102.6(4.0)$ & $105.4(4.4)$ \\
\hline $\mathrm{tPV}(\mathrm{ms})$ & $0.749(0.11)$ & $333(15)$ & $334(16)$ & $326(14)$ & $328(14)$ & $330(15)$ & $326(15)$ \\
\hline PGA (cm) & $0.000(75.29)$ & $12.1(0.2)$ & $11.3(0.2)$ & $12.1(0.2)$ & $11.3(0.2)$ & $12.0(0.1)$ & $11.2(0.2)$ \\
\hline $\mathrm{tPGA}(\mathrm{ms})$ & $0.000(56.12)$ & $679(34)$ & $756(36)$ & $666(30)$ & $746(32)$ & $673(33)$ & $746(35)$ \\
\hline
\end{tabular}

${ }^{a} p$ values and inferential statistics ( $F$ values are within parentheses) for the factor "object slant" (following control stimulation) over each kinematic parameter.

${ }^{b}$ Descriptive statistics (mean with SEM within parentheses) as a function of TMS site and object slant. The effects of TMS site (three levels: vertex as a control site, alPS, and sP0S), object slant (two levels: vertical, horizontal), and their interaction were tested on each kinematic parameter using a univariate repeated measures ANOVA with 1 degree of freedom for the test, and 22 for the residual. Compared with control, TMS reaction times were reduced after either alPS or sPOS stimulation, but this effect was only significant following TMS over alPS. For the remaining kinematic parameters only the factor object slant revealed some significant results ( $p<0.05$ ), but not the factor TMS site, nor the interaction of slant $\times$ TMS site.

that, in the current experimental setup, grasping a horizontally oriented object entails the satisfaction of additional spatial constraints on the index finger, increasing movement complexity with respect to grasping a vertically oriented object. It can also be seen that, in the second half of the movement, the variability of the trajectories of both fingers and hand was larger when grasping horizontally oriented objects (index finger: between 52 and 75\% of path length, $p=0.019$; thumb: $69-97 \%, p<0.001$; hand: $21-100 \%, p<0.001$ ) (Fig. $2 C-E$ ). This effect occurs in midflight, when the fingers and the hand travel over the object. During this phase, those effectors reach higher velocity than when grasping vertically oriented objects (Fig. $3 E$ ), and their position is not spatially restricted as when the fingers need to be in contact with the object. These conditions lead to a generalized increase in spatial variability of the trajectories of both fingers and hand during this stage of the movement. This effect is consistent with the notion that, when grasping a horizontally oriented object, coordinating fingers and hand is more complex. Finally, in contrast to the thumb and index finger, the variability of the hand remains larger when grasping horizontally oriented objects even in the final $5 \%$ of the movement, in accordance with the fact that the back of the hand was not spatially restricted even in the final phase of the movement.

Other kinematic parameters corroborated the inferences drawn from the spatial variability of the movements of the hand, thumb, and index finger (Table 1; Fig. 3) (main effect of slant within the control session). The hand traveled a longer path at a slightly higher average speed, resulting in longer movement durations (MT, TL) (Fig. 3B,C). The peak velocity was slightly higher when grasping horizontal compared with vertical objects, but occurred at the same time point after movement onset (PV) (Fig. 3D). Importantly, the velocity of the fingers had similar 


\section{EEG effects of high sensorimotor demands (object slant)}
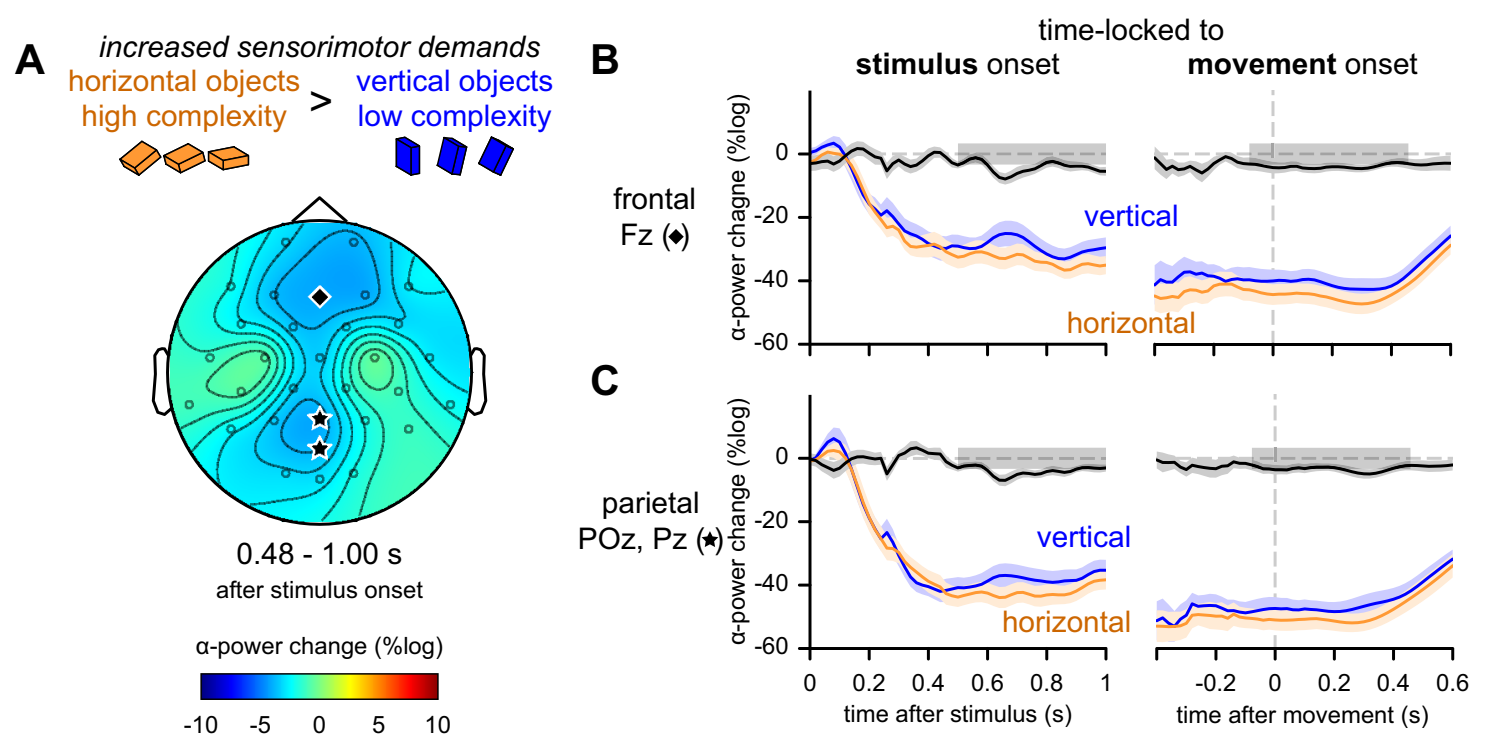

Figure 4. EEG effects of increased sensorimotor demands (object slant) during control TMS. A, Differential topography of power in the alpha-band after control stimulation as a factor of slant in the significant time window (time-locked to stimulus onset; $p<0.05$ corrected for multiple-comparisons). Alpha-suppression is stronger during preparation and execution of a grasping movement toward horizontally slanted objects. $\boldsymbol{B}$, Changes in oscillatory power over time in electrode $\mathrm{Fz}$ (indicated with a diamond in $\boldsymbol{A}$ ) shown separately for trials with vertical (blue) and horizontal objects (orange) and their difference (black), time-locked to the stimulus (left) or to movement onset (right). Significant time windows are indicated with gray boxes ([480 1000] ms after stimulus onset, left panels; [ -60440$]$ ms relative to movement onset, right panels). $\boldsymbol{C}$, Changes in average power of electrodes $\mathrm{POz}$ and $\mathrm{Pz}$ (indicated with stars in $\boldsymbol{A}$ ). Same conventions as in $\boldsymbol{B}$.

dynamics as the variability of the index finger trajectory, namely being significantly higher when grasping horizontal compared with vertical objects in the first three quartiles of the movement $\left(\mu \mathrm{TV}: F_{(1,22)}=14.69, p<0.001\right)$ (Fig. $\left.3 E\right)$, although being significantly lower in the last quartile ( $\mu \mathrm{AV}: F_{(1,22)}=6.42, p=$ 0.019 ) (Fig. 3F). In contrast to these widespread effects on the grasping kinematics, object slant did not influence the duration of movement planning (RT: $F_{(1,22)}=0.26, p=0.615$ ) (Fig. $3 A$ ). We also observed that with increasing slant from vertical to horizontal the peak grip aperture became smaller and occurred later (PGA and tPGA) (Table 1; Fig. 3G,H). This finding has been consistently reported in the literature (Mamassian, 1997; Desmurget et al., 1998; van Bergen et al., 2007). When grasping vertical objects the hand palm is facing the frontal surface of the object, and the peak grip aperture is reached by opening both the index finger and thumb. However, when grasping horizontally slanted objects, the hand palm is not fully rotated with the object to minimize effort (otherwise the hand would need to be lifted directly above the object). The remaining rotation of the grip is achieved by keeping the thumb closer to the hand palm, and the index finger fully extended. Accordingly, the peak grip aperture is limited when grasping more horizontally oriented objects. This biomechanical constraint overshadows any potential influence of changes in planning uncertainty on the peak grip aperture (Jeannerod, 1986).

\section{Cerebral effects of grasping a slanted object: increased sensorimotor demands enhance dorsomedial alpha-suppression}

Planning and executing the grasping movement led to strong suppression of ongoing low-frequency oscillations (alpha, 8-12 $\mathrm{Hz}$, over parieto-occipital sensors; beta, $18-24 \mathrm{~Hz}$, over frontoparietal sensors) (Verhagen et al., 2012), a well known index of increased computational load (Neuper et al., 2006; Jensen and Mazaheri, 2010).
As object slant increased from vertical to horizontal, movement complexity increased, and alpha-power over dorsomedial parietal and frontal areas was suppressed even further $(p=0.004$; Fig. $4 A$ ). Importantly, this additional alpha-suppression, related to increased sensorimotor complexity, arose just before movement onset and persisted during the first half of the movement, when vision of the hand and object was prevented (Fig. 4B,C).

The enhanced alpha-suppression in trials with the object in a more horizontal orientation (from here on labeled "sensorimotor alpha-suppression") was not modulated by the type of depth information allowed by vision (binocular or monocular: post hoc, $p=0.65$ ). In general, we found no significant differences in oscillatory power between monocular and binocular trials or significant interactions between object vision (monocular, binocular) and TMS site. This indicates that the changes in sensorimotor alpha-suppression are purely a consequence of the requested motor output, and not driven by the information value of the visual input, or the type of depth information (Knill, 2005).

However, the lack of differences in oscillatory power between monocular and binocular trials does not imply a discrepancy with well known effects of visual input on event-related potentials (ERP) (Campbell and Green, 1965; Perry et al., 1968). In fact, a post hoc time-locked ERP analysis indicated that visually evoked potentials (N70-P100-N140, relative to stimulus onset) had a higher peak-to-peak amplitude during binocular compared with monocular vision, regardless of object orientation and stimulation site $(p<0.001)$. This observation matches previous findings of changes in both EEG ERPs and fMRI BOLD signals originating from early visual cortex (Campbell and Green, 1965; Perry et al., 1968; Verhagen et al., 2008). Importantly, this early effect of object vision occurred before the delivery of the TMS pulse, and it did not interact with the other experimental factors, or with the induced oscillatory power effects following the TMS delivery. 


\section{EEG effects of high sensorimotor demands (TMS $x$ object slant)}

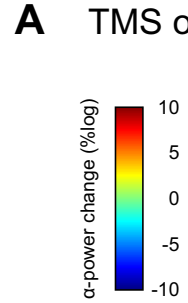

B

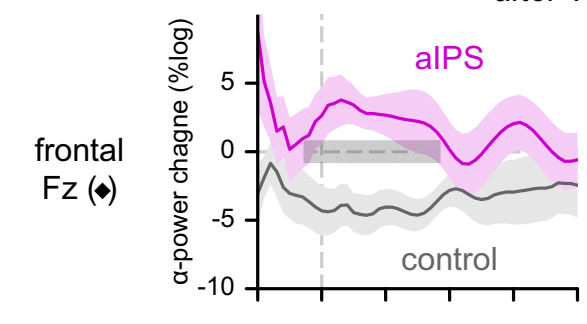

C
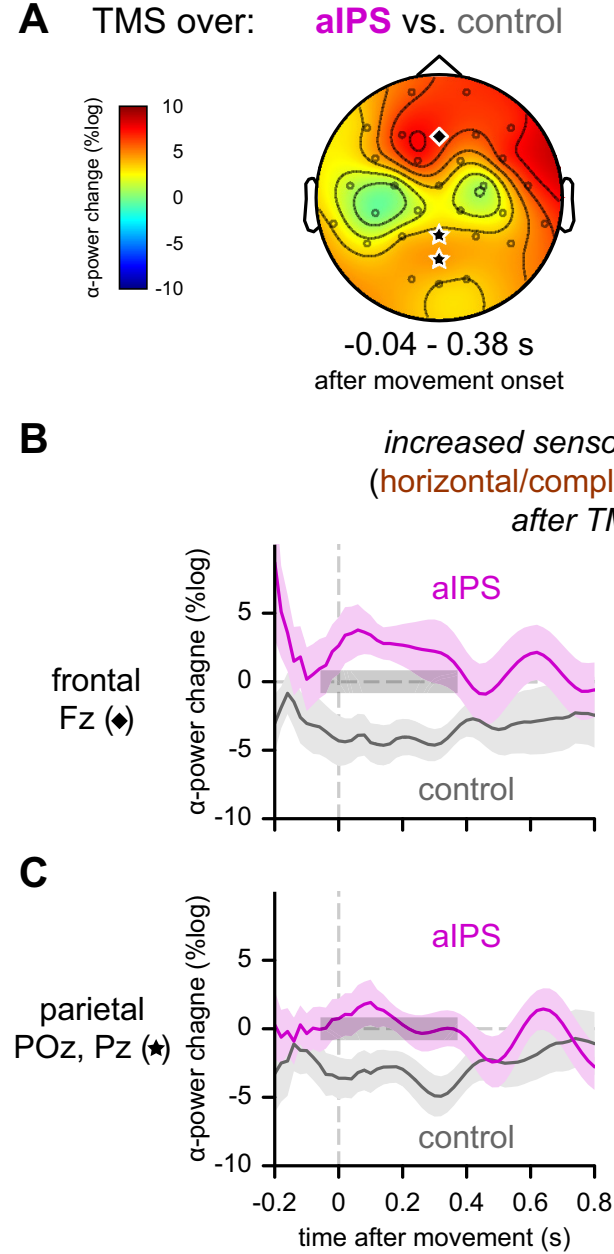

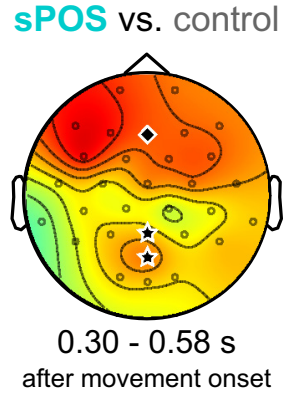

after movement onset increased sensorimotor demands

(horizontal/complex > vertical/easy) after TMS over:
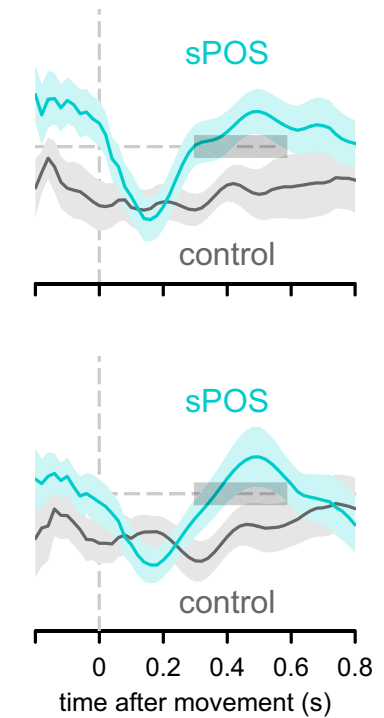

Figure 5. Differential EEG effects of increased sensorimotor demands (object slant) as a function of TMS site. Left, alPS versus control. Right, $s$ POS versus control. $A$, Differential topographies of alpha power representing significant modulation of the effect of slant (grasping horizontal $>$ vertical objects) as a function of stimulation site. The enhanced alpha-suppression after control stimulation for horizontal objects is disrupted both by aIPS and sPOS stimulation, but in a different time-window (alPS: [ -40380 ] ms; SPOS: [300 580] ms relative to movement onset). $\boldsymbol{B}$, C, The differential effects of slant are plotted separately following control (left and right panels; gray), alPS (left panels; magenta), and sPOS stimulation (right panels; cyan) for electrode $\mathrm{Fz}(\boldsymbol{B})$ and the average of electrodes $\mathrm{POz}$ and $\mathrm{Pz}(\mathbf{C}$. Significant time windows are indicated with gray boxes.

\section{Behavioral consequences of TMS perturbation over aIPS and sPOS}

The TMS perturbations had limited effects on task performance. In fact, the factor TMS-site did not reveal a significant effect on any of the behavioral parameters (all $p>0.1$ ). However, a direct post hoc comparison (uncorrected for multiple-comparisons) between control TMS and TMS over aIPS showed that subjects started moving $55 \mathrm{~ms}$ sooner after stimulus presentation following TMS over aIPS, irrespectively of object slant or viewing conditions $(F=4.87 ; p=0.038)$ (Fig. $3 A)$. In a similar direct comparison, stimulation of sPOS had no significant behavioral effect. Despite using low-intensity stimulation and earplugs, sound levels were not matched between stimulation sites given that the distance between coil and ear differed. More precisely, the aIPS site was closest to the ear and the vertex site farthest. Because the effect of stimulation on reaction times did not interact with the task manipulations of object slant and vision, it is likely that this reaction time facilitation could be a result of a nonspecific intersensory facilitation or an acoustic startle effect
(Nickerson, 1973; Valls-Sole et al., 1999; de Graaf and Sack, 2011). Importantly, we also did not observe any behavioral interaction effects of TMS site with object slant (Table 1; Fig. 3), indicating that the low-intensity TMS pulses used in this study (100\% AMT) did not perturb the sensorimotor system outside its physiological range, avoiding uncontrolled by-products of strategic changes in behavior.

\section{Cerebral consequences of TMS perturbation over aIPS and sPOS: functional dependencies between dorsolateral and dorsomedial circuits} To probe the functional dependencies between the dorsolateral and dorsomedial parietofrontal channels we used TMS to interfere with processing in aIPS and sPOS and tracked the consequences on cerebral computations supporting action planning and execution. Accordingly, we tested where and when sensorimotor alpha-suppression (Fig. 4) differed as a function of TMS site. We found that compared with control stimulation, TMS of both aIPS and sPOS diminished the sensorimotor alpha-suppression over medial parietofrontal cortex (aIPS: $p=0.006$; sPOS: $p=0.017$ ) (Fig. $5 A$ ).

These interactions of TMS site with sensorimotor alpha-suppression could be further specified in both topography and temporal dynamics. The spatial distribution of the perturbation effects evoked by aIPS and sPOS TMS overlapped with the topography of unaffected sensorimotor alpha-suppression evoked by vertex TMS (Figs. $4 A, 5 A$ ). The perturbation effects were observed on top of the already strong low-frequency oscillatory suppression of these regions during action planning and execution (Fig. 4B,C) (Verhagen et al., 2012). Importantly, the timing of the effects differed: aIPS stimulation altered the sensorimotor alpha-suppression early during movement performance ( $[-40380]$ ms relative to movement onset) (Fig. $5 B, C$, left panels), whereas sPOS stimulation induced a late alteration ([300 580] ms after movement onset) (Fig. $5 B, C$, right panels). Stimulation over the two parietal sites also differed significantly from each other early after movement onset ( $[80$ 260] ms after movement onset; $p=0.025$ ). These findings indicate that aIPS and sPOS contribute to the same sensorimotor processes, but are critically different in the timing of their contributions.

\section{Dorsolateral alpha-suppression after sPOS perturbation}

Above, we described the effects of movement complexity as a function of object slant and the interaction with TMS site. In addition, we observed one important general effect of TMS site, regardless of object slant. TMS over sPOS, compared with TMS over vertex, led to a sustained stronger suppression in the alpha frequency band over left anterior lateral parietal cortex. This sup- 
pression was localized around electrode CP5 (putatively located over the superior marginal gyrus in the inferior parietal lobule) (Koessler et al., 2009) and continued throughout movement planning and the early stages of execution ( $p=0.014$ ) (Fig. $6 A, B)$. In contrast, no main effect of TMS over aIPS was observed.

A priori, TMS perturbation of a region involved in the task, sPOS in this case, could be expected to cause a local reduction in low-frequency (alpha-band) power, reflecting disrupted neural computations. Here, instead, we observed a distant enhancement of alpha power that transcended the contribution of sPOS in both time and function. This effect was not accompanied by a change in kinematic behavior (Table 1). Together, these findings suggest that the increased alphaband suppression in anterior lateral parietal cortex might have a general compensatory function following perturbation of sPOS by TMS. The alphasuppression evoked by TMS over sPOS was not modulated by the type of vision allowed (binocular or monocular: post hoc, $p=0.96)$.

\section{Discussion}

This study assessed temporal and functional dependencies between parietofrontal circuits supporting goal-directed action, focusing on the relation between aIPS and sPOS contributions. Subjects grasped an object oriented at different slants, while maintaining constant object position, size, and shape. This manipulation varied movement complexity and consequently the demands on sensorimotor processing. There are three main results. First, when subjects grasped horizontally oriented objects and sensorimotor complexity increased, there was enhanced suppression of oscillatory power in the alpha-band over dorsomedial parietofrontal areas (in short, sensorimotor alpha-suppression), starting before movement onset and continuing during movement execution. This finding indicates that grasping parameters are specified in the dorsomedial parietofrontal circuit, just before movement onset. Second, disturbing either aIPS or sPOS during movement planning with single-pulse TMS had the same electrophysiological consequences, namely a disruption of the sensorimotor alpha-suppression over dorsomedial parietofrontal areas, with one important difference in the temporal dynamics. TMS over aIPS affected processing in the dorsomedial circuit considerably earlier than TMS over sPOS. This finding indicates that processing in aIPS and sPOS converges on the same dorsomedial sensorimotor computations, but with aIPS contributions preceding those of sPOS. Third, applying TMS over sPOS enhanced computational load (alpha-suppression) over dorsolateral parietal regions, from early planning until movement execution. Given that these cerebral effects occurred in the context of unaltered behavior, and adequate sensitivity for capturing behavioral consequences of the TMS intervention (Verhagen et al., 2012), we infer that dorsolateral parietal regions compensate for a transient sPOS disruption. Together, these observations indicate that both dorsomedial and dorsolateral parietofrontal circuits support the same functional components of grasping, in the context of a serial temporal dependency of dorsomedial processing on aIPS.

\section{The dorsomedial parietofrontal circuit specifies grasping motor parameters shortly before movement onset}

We have previously shown that planning and executing a grasping movement led to strong suppression of oscillatory alpha power over occipital, parietal, and frontal sensors (Verhagen et al., 2012). Here, we report that alpha-suppression over dorsomedial parietofrontal regions changes as a function of grasp complexity: the suppression becomes stronger when the slant of the grasped object increases (Fig. 4). This effect is relevant because, in the current experimental setting, grasping horizontally slanted objects increases the computational load of the grasping movement. The object acts as an obstacle for seeing and reaching the intended digit end-points, and it requires the coordination of multiple joints along a longer path (Gentilucci et al., 1996; Ramnani et al., 2001), resulting in slant-related changes in trajectory variability (Fig. 2). The scalp topography of the sensorimotor demand-related alpha-suppression matches the spatial distribution of increased BOLD activity evoked by grasping objects at increasing slants, previously found to be centered on sPOS and PMd (Verhagen et al., 2008). These kinematic and electrophysiological findings provide evidence that sPOS deals with the control of hand and fingers during grasping, rather than the transport of the hand only (Cavina-Pratesi et al., 2010; Gallivan et al., 2011). Crucially, the dorsomedial sensorimotor alphasuppression started shortly before movement onset, suggesting that this element of the grasping movement is incorporated in the motor plan at a late stage. This observation fits with the timing of visuomotor neurons recorded from area V6A in macaques that 
sharply increase in firing rate just before movement onset and continue to fire during movement execution (Galletti et al., 2003; Fattori et al., 2009). Together, these findings indicate that the dorsomedial parietofrontal circuit specifies motor parameters controlling arm, wrist, and finger movements with respect to object configuration, and that those parameters are specified just before movement onset.

\section{aIPS supports grasping parameterization before $\mathrm{SPOS}$}

Single-pulse TMS interference over aIPS or sPOS during grasp planning reduced sensorimotor alpha-suppression over dorsomedial parietofrontal regions (Fig. $5 A$ ). This effect was strongest when TMS was delivered while subjects planned to grasp horizontally slanted objects, and its characteristics changed according to the site of the TMS intervention. Sensorimotor alphasuppression occurring around movement onset was disturbed by aIPS stimulation, whereas sPOS stimulation reduced alphasuppression later during the movement. Two inferences can be drawn from these observations. First, the spatial and spectral overlap of the TMS effects indicate that disturbing aIPS and sPOS has the same neural consequences during grasp planning, suggesting that these areas support the computation of similar grasp parameters. This inference fits with empirical findings reporting similar neuronal properties of these regions (Murata et al., 2000; Fattori et al., 2009). Second, the different temporal dynamics of the TMS effects indicates that sPOS computations follow information processing in aIPS early during planning. We infer that the dorsomedial circuit specifies motor parameters on the basis of object knowledge already processed in the dorsolateral stream, which in turn is informed by information elaborated in the ventral visual stream (Verhagen et al., 2008, 2012). The effects on dorsomedial alpha power were independent of the available visual cues (binocular, monocular) suggesting that sPOS computations might be functionally closer to the motor output than to the visual input, in line with the observation that the macaque homolog of this parietal region shows a relative abundance of motor over visual neurons (Gamberini et al., 2011).

The present data indicate that the two anatomically distinct parietofrontal circuits (Rizzolatti and Matelli, 2003) ignore the phenomenological border between motor planning and online control (Glover, 2004). Instead, a hierarchical organization between dorsolateral and dorsomedial circuits exists during motor planning, and it continues to operate during movement execution. We suggest that grasp computations, and not grasp timing, can be divided in two levels. The first level, impinging preferentially on the dorsolateral circuit, constructs an object-based action prior (Verhagen et al., 2012). The second level, impinging preferentially on the dorsomedial circuit, transforms currently available visuospatial evidence, in the context of the priors provided by the first level. In this framework, when object affordances change (Tunik et al., 2005), then dorsolateral computations become critical for online control; when extrinsic visuospatial parameters change (Desmurget et al., 1999), then dorsomedial computations are additionally required to adjust the grasping movement.

\section{The dorsolateral circuit can compensate for sPOS perturbation}

When sPOS activity was disturbed with TMS during motor preparation, there was a sustained increase in alpha-suppression over a left inferior parietal area, starting early during movement planning and continuing well into the execution phase (Fig. 6). This enhanced alpha-suppression over inferior parietal cortex was not a consequence of changes in grasping behavior, and emerged at a site and at a time remote from the TMS interference. Differently from the dorsomedial sensorimotor alpha-suppression effect discussed in the previous sections (Figs. 4, 5), the enhanced alphasuppression over inferior parietal cortex was a main effect of stimulation site (TMS over sPOS vs TMS over vertex), and it started early during planning. The temporal and spatial characteristics of the enhanced alpha-suppression suggest that the dorsolateral circuit can compensate for a transient sPOS perturbation. This observation fits nicely with the grasping behavior of optic ataxia patients with bilateral lesions of the superior parietooccipital cortex (Karnath and Perenin, 2005). These patients are impaired when asked to grasp a cylindrical object they have not encountered before (Jeannerod, 1986). However, they can skillfully grasp familiar objects of the same size and shape, for example, a tube of lipstick (Jeannerod et al., 1994). If the novel objects are presented repeatedly, the initially impaired hand shaping improves with increasing familiarity (Milner et al., 2001). The behavior of these patients suggests that object knowledge and previous experience, presumably processed in the dorsolateral circuit on the basis of computations performed in the ventral visual stream (Verhagen et al., 2012), can be used for structuring grasping movements when the dorsomedial stream is impaired. Accordingly, we speculate that the putative compensatory activity of inferior parietal regions we observed in this study could exploit the ability of the dorsolateral circuit to generate priors that constrain the grasp plan on the basis of abstract object knowledge (Arbib, 1985; Johnson and Grafton, 2003; Verhagen et al., 2012).

\section{Conclusion}

By measuring the behavioral and electrophysiological consequences of transient perturbation of aIPS and sPOS we have shown that the computations of both parietal areas converge on the same dorsomedial parietofrontal circuit, with aIPS contributions preceding those of sPOS. Furthermore, perturbation of sPOS was compensated by inferior parietal activity. These observations define the temporal and functional characteristics of aIPS and sPOS contributions to goal-directed grasping movements, and suggest a novel mechanism for the organization of these movements. First, aIPS computes an initial structure of the motor plan on the basis of object knowledge gathered from both dorsal and ventral visual streams (Verhagen et al., 2008, 2012). Second, dorsomedial parietofrontal regions use this movement structure as a prior for guiding the accurate parameterization of the movement in space. This mechanism also offers a reinterpretation of previous accounts of the organization of goal-oriented movements (Kandel et al., 2000; Glover, 2004; Culham et al., 2006). Planning versus online control and reaching versus grasping might be phenomenological instances of the underlying hierarchical organization of the dorsolateral and dorsomedial circuits, reflecting their temporal dependencies and the different levels of abstraction at which those circuits operate.

\section{References}

Allen EA, Pasley BN, Duong T, Freeman RD (2007) Transcranial magnetic stimulation elicits coupled neural and hemodynamic consequences. Science 317:1918-1921. CrossRef Medline

Arbib MA (1985) Schemas for the temporal organization of behaviour. Hum Neurobiol 4:63-72. Medline

Borra E, Belmalih A, Calzavara R, Gerbella M, Murata A, Rozzi S, Luppino G (2008) Cortical connections of the macaque anterior intraparietal (AIP) area. Cereb Cortex 18:1094-1111. CrossRef Medline

Brouwer AM, Franz VH, Gegenfurtner KR (2009) Differences in fixations 
between grasping and viewing objects. J Vis 9(1):18 1-24. CrossRef Medline

Campbell FW, Green DG (1965) Monocular versus binocular visual acuity. Nature 208:191-192. CrossRef Medline

Cavina-Pratesi C, Monaco S, Fattori P, Galletti C, McAdam TD, Quinlan DJ, Goodale MA, Culham JC (2010) Functional magnetic resonance imaging reveals the neural substrates of arm transport and grip formation in reach-to-grasp actions in humans. J Neurosci 30:10306-10323. CrossRef Medline

Chatrian GE, Lettich E, Nelson PL (1985) Ten percent electrode system for topographic studies of spontaneous and evoked EEG activity. Am J EEG Technol 25:83-92.

Culham JC, Cavina-Pratesi C, Singhal A (2006) The role of parietal cortex in visuomotor control: what have we learned from neuroimaging? Neuropsychologia 44:2668-2684. CrossRef Medline

Davare M, Andres M, Cosnard G, Thonnard JL, Olivier E (2006) Dissociating the role of ventral and dorsal premotor cortex in precision grasping. J Neurosci 26:2260-2268. CrossRef Medline

de GraafTA, Sack AT (2011) Null results in TMS: from absence of evidence to evidence of absence. Neurosci Biobehav Rev 35:871-877. CrossRef Medline

Desmurget M, Gréa H, Prablanc C (1998) Final posture of the upper limb depends on the initial position of the hand during prehension movements. Exp Brain Res 119:511-516. CrossRef Medline

Desmurget M, Epstein CM, Turner RS, Prablanc C, Alexander GE, Grafton ST (1999) Role of the posterior parietal cortex in updating reaching movements to a visual target. Nat Neurosci 2:563-567. CrossRef Medline

Fattori P, Breveglieri R, Marzocchi N, Filippini D, Bosco A, Galletti C (2009) Hand orientation during reach-to-grasp movements modulates neuronal activity in the medial posterior parietal area V6A. J Neurosci 29:19281936. CrossRef Medline

Fattori P, Raos V, Breveglieri R, Bosco A, Marzocchi N, Galletti C (2010) The dorsomedial pathway is not just for reaching: grasping neurons in the medial parieto-occipital cortex of the macaque monkey. J Neurosci 30: 342-349. CrossRef Medline

Galletti C, Kutz DF, Gamberini M, Breveglieri R, Fattori P (2003) Role of the medial parieto-occipital cortex in the control of reaching and grasping movements. Exp Brain Res 153:158-170. CrossRef Medline

Gallivan JP, McLean A, Culham JC (2011) Neuroimaging reveals enhanced activation in a reach-selective brain area for objects located within participants' typical hand workspaces. Neuropsychologia 49:3710-3721. CrossRef Medline

Gamberini M, Passarelli L, Fattori P, Zucchelli M, Bakola S, Luppino G, Galletti C (2009) Cortical connections of the visuomotor parietooccipital area V6Ad of the macaque monkey. J Comp Neurol 513:622-642. CrossRef Medline

Gamberini M, Galletti C, Bosco A, Breveglieri R, Fattori P (2011) Is the medial posterior parietal area V6A a single functional area? J Neurosci 31:5145-5157. CrossRef Medline

Gentilucci M, Daprati E, Gangitano M, Saetti MC, Toni I (1996) On orienting the hand to reach and grasp an object. Neuroreport 7:589-592. CrossRef Medline

Gerbella M, Belmalih A, Borra E, Rozzi S, Luppino G (2011) Cortical connections of the anterior (F5a) subdivision of the macaque ventral premotor area F5. Brain Struct Funct 216:43-65. CrossRef Medline

Gharbawie OA, Stepniewska I, Kaas JH (2011) Cortical connections of functional zones in posterior parietal cortex and frontal cortex motor regions in new world monkeys. Cereb Cortex 21:1981-2002. CrossRef Medline

Glover S (2004) Separate visual representations in the planning and control of action. Behav Brain Sci 27:3-24; discussion 24-78. CrossRef Medline

Grafton ST (2010) The cognitive neuroscience of prehension: recent developments. Exp Brain Res 204:475-491. CrossRef Medline

Ilmoniemi RJ, Kicić D (2010) Methodology for combined TMS and EEG. Brain Topogr 22:233-248. CrossRef Medline

Jasper HH (1958) The ten-twenty electrode system of the international federation. Electroencephalogr Clin Neurophysiol 10:371-375.

Jeannerod M (1984) The timing of natural prehension movements. J Mot Behav 16:235-254. Medline

Jeannerod M (1986) Mechanisms of visuomotor coordination: a study in normal and brain-damaged subjects. Neuropsychologia 24:41-78. CrossRef Medline
Jeannerod M, Decety J, Michel F (1994) Impairment of grasping movements following a bilateral posterior parietal lesion. Neuropsychologia 32:369-380. CrossRef Medline

Jeannerod M, Arbib MA, Rizzolatti G, Sakata H (1995) Grasping objects: the cortical mechanisms of visuomotor transformation. Trends Neurosci 18:314-320. CrossRef Medline

Jensen O, Mazaheri A (2010) Shaping functional architecture by oscillatory alpha activity: gating by inhibition. Front Hum Neurosci 4:186. CrossRef Medline

Johnson SH, Grafton ST (2003) From "acting on" to "acting with": the functional anatomy of object-oriented action schemata. Prog Brain Res 142:127-139. CrossRef Medline

Kandel ER, Schwartz JH, Jessell TM (2000) Principles of neural science, Ed 4. New York, London: McGraw-Hill.

Karnath HO, Perenin MT (2005) Cortical control of visually guided reaching: evidence from patients with optic ataxia. Cereb Cortex 15:1561-1569. CrossRef Medline

Knill DC (2005) Reaching for visual cues to depth: the brain combines depth cues differently for motor control and perception. J Vis 5(2):2 103-115. CrossRef Medline

Koessler L, Maillard L, Benhadid A, Vignal JP, Felblinger J, Vespignani H, Braun M (2009) Automated cortical projection of EEG sensors: anatomical correlation via the international $10-10$ system. Neuroimage 46 : 64-72. CrossRef Medline

Korhonen RJ, Hernandez-Pavon JC, Metsomaa J, Mäki H, Ilmoniemi RJ, Sarvas J (2011) Removal of large muscle artifacts from transcranial magnetic stimulation-evoked EEG by independent component analysis. Med Biol Eng Comput 49:397-407. CrossRef Medline

Lee TW, Girolami M, Sejnowski TJ (1999) Independent component analysis using an extended infomax algorithm for mixed subgaussian and supergaussian sources. Neural Comput 11:417-441. CrossRef Medline

Liu D, Todorov E (2007) Evidence for the flexible sensorimotor strategies predicted by optimal feedback control. J Neurosci 27:9354-9368. CrossRef Medline

Makeig S, Debener S, Onton J, Delorme A (2004) Mining event-related brain dynamics. Trends Cogn Sci 8:204-210. CrossRef Medline

Mamassian P (1997) Prehension of objects oriented in three-dimensional space. Exp Brain Res 114:235-245. CrossRef Medline

Maris E, Oostenveld R (2007) Nonparametric statistical testing of EEG- and MEG-data. J Neurosci Methods 164:177-190. CrossRef Medline

McIntyre J, Stratta F, Lacquaniti F (1998) Short-term memory for reaching to visual targets: psychophysical evidence for body-centered reference frames. J Neurosci 18:8423-8435. Medline

Milner AD, Dijkerman HC, Pisella L, McIntosh RD, Tilikete C, Vighetto A, Rossetti Y (2001) Grasping the past: delay can improve visuomotor performance. Curr Biol 11:1896-1901. CrossRef Medline

Murata A, Gallese V, Luppino G, Kaseda M, Sakata H (2000) Selectivity for the shape, size, and orientation of objects for grasping in neurons of monkey parietal area AIP. J Neurophysiol 83:2580-2601. Medline

Neuper C, Wörtz M, Pfurtscheller G (2006) ERD/ERS patterns reflecting sensorimotor activation and deactivation. Prog Brain Res 159:211-222. CrossRef Medline

Nickerson RS (1973) Intersensory facilitation of reaction time: energy summation or preparation enhancement? Psychol Rev 80:489-509. CrossRef Medline

Oldfield RC (1971) The assessment and analysis of handedness: the Edinburgh inventory. Neuropsychologia 9:97-113. CrossRef Medline

Oostenveld R, Fries P, Maris E, Schoffelen JM (2011) FieldTrip: open source software for advanced analysis of MEG, EEG, and invasive electrophysiological data. Comput Intell Neurosci 2011:156869. CrossRef Medline

Perry NW Jr, Childers DG, McCoy JG (1968) Binocular addition of the visual evoked response at different cortical locations. Vision Res 8:567573. CrossRef Medline

Pisella L, Gréa H, Tilikete C, Vighetto A, Desmurget M, Rode G, Boisson D, Rossetti Y (2000) An "automatic pilot" for the hand in human posterior parietal cortex: toward reinterpreting optic ataxia. Nat Neurosci 3:729 736. CrossRef Medline

Ramnani N, Toni I, Passingham RE, Haggard P (2001) The cerebellum and parietal cortex play a specific role in coordination: a PET study. Neuroimage 14:899-911. CrossRef Medline

Rizzolatti G, Matelli M (2003) Two different streams form the dorsal visual 
system: anatomy and functions. Exp Brain Res 153:146-157. CrossRef Medline

Rossini PM, Barker AT, Berardelli A, Caramia MD, Caruso G, Cracco RQ, Dimitrijević MR, Hallett M, Katayama Y, Lucking CH, et al. (1994) Non-invasive electrical and magnetic stimulation of the brain, spinal cord and roots: basic principles and procedures for routine clinical application. Report of an IFCN committee. Electroencephalogr Clin Neurophysiol 91:79-92. CrossRef Medline

Sack AT, Cohen Kadosh R, Schuhmann T, Moerel M, Walsh V, Goebel R (2009) Optimizing functional accuracy of TMS in cognitive studies: a comparison of methods. J Cogn Neurosci 21:207-221. CrossRef Medline

Schot WD, Brenner E, Smeets JB (2010) Robust movement segmentation by combining multiple sources of information. J Neurosci Methods 187:147-155. CrossRef Medline

Taubert M, Dafotakis M, Sparing R, Eickhoff S, Leuchte S, Fink GR, Nowak DA (2010) Inhibition of the anterior intraparietal area and the dorsal premotor cortex interfere with arbitrary visuo-motor mapping. Clin Neurophysiol 121:408-413. CrossRef Medline

Tunik E, Frey SH, Grafton ST (2005) Virtual lesions of the anterior intraparietal area disrupt goal-dependent on-line adjustments of grasp. Nat Neurosci 8:505-511. CrossRef Medline
Tunik E, Ortigue S, Adamovich SV, Grafton ST (2008) Differential recruitment of anterior intraparietal sulcus and superior parietal lobule during visually guided grasping revealed by electrical neuroimaging. J Neurosci 28:13615-13620. CrossRef Medline

Valls-Sole J, Rothwell JC, Goulart F, Cossu G, Munoz E (1999) Patterned ballistic movements triggered by a startle in healthy humans. J Physiol 516:931-938.

van Bergen E, van Swieten LM, Williams JH, Mon-Williams M (2007) The effect of orientation on prehension movement time. Exp Brain Res 178: 180-193. CrossRef Medline

Verhagen L, Dijkerman HC, Grol MJ, Toni I (2008) Perceptuo-motor interactions during prehension movements. J Neurosci 28:4726-4735. CrossRef Medline

Verhagen L, Dijkerman HC, Medendorp WP, Toni I (2012) Cortical dynamics of sensorimotor integration during grasp planning. J Neurosci 32:4508-4519. CrossRef Medline

Vesia M, Crawford JD (2012) Specialization of reach function in human posterior parietal cortex. Exp Brain Res 221:1-18. CrossRef Medline 